\title{
Beobachtungen über das Verhalten der Seezungenbrut
}

\author{
HaRALD Rosenthal \\ Institut fïr Hydrobiologie wnd Fischereiwissenschaft der Universität, Hamburg
}

\begin{abstract}
Observations on the behavior of sole fry. In spring 1965 eggs of the sole Solea solea L. were hatched in the Marine Station of the Biologische Anstalt Helgoland (island Helgoland, North Sea). The newly hatched larvae were raised under laboratory conditions and their behavior, food relationships and swimming performance studied. Yolk-sac larvae swim rather clumsily and often turn around their body axis. Their main swimming direction is downward, compensating for a tendency toward buoyancy. At the time of their first food intake, most larvae stay close to the bottom and search as substrate feeders for food. In free water, hungry young larvae occasionally try to catch food but obviously lack appropriate aiming techniques. Nine to ten days after the first food intake, the symmetrical larvae begin to rest temporarily sidewise on the ground; no body side is preferred at this stage. Digging into the substratum (sand) was not observed before metamorphosis. On the basis of movie pictures, yolk-sac larvale (at the time of their first food intake) swim on an average 6 to $9 \mathrm{~mm}$ per second (200 to $230 \mathrm{~m}$ per 12 hour day). Tow ard the end of the yolk-sac stage the larvae are active during up to $75 \%$ of the observation period. Five days after hatching, unfed larvae, kept at $15^{\circ} \mathrm{C}$, reduce their swimming activity to about $50 \%$ of the normal level; after 8 days swimming performance is reduced below $4108 \mathrm{~m}$. On the basis of our laboratory observations, the pelagic phase is restricted mainly to the yolk-sac phase.
\end{abstract}

\section{EINLEITUNG}

Im Frühjahr 1965 wurden in der Meeresstation der Biologischen Anstalt Helgoland Erbrütungs- und Aufzuchtversuche an Seezungen (Solea solea L.) durchgeführt. In seiner Darstellung der Erbrütungs- und Hälterungstechnik hat FLücHTER (1965) einige Verhaltensweisen der jungen Brut mitgeteilt. In Ergänzung dazu soll in der vorliegenden Arbeit über Verhaltensanalysen an Larven verschiedenen Alters berichtet werden, wie sie erstmals unter kontrollierten Laborbedingungen möglich waren.

Uber die Naturgeschichte der Seezungenbrut ist bisher relativ wenig bekannt. Während die Dottersacklarven in jedem Früjahr zahlreich gefangen werden konnten, blieb die Suche nach den älteren Larven und jüngsten Bodenstadien meistens erfolglos. Ehrenbaum $(1907,1914)$ und Cunningham $(1890)$ beobachteten nur selten das Auftreten der bereits metamorphisierten, etwa 12 bis $15 \mathrm{~mm}$ langen Jungfische. Die aus der Literatur bekannten Fangdaten stammen ausschließlich aus flachen küstennahen Gebieten. Man war der Ansicht, daß die Larven der Seezunge als reine Planktonfresser pelagisch leben und erst nach der Metamorphose zur benthischen Lebensweise übergehen. Fabre-Domergue \& Bietrix (1905) nehmen nach ihren Beobachtungen eine pelagische Lebensweise von 4 bis 6 Wochen an. Die größeren Jungtiere von 2 bis $4 \mathrm{~cm}$ 
Totallänge an werden bereits im Watt und an den Küsten der Nordsee gefangen. In der Deutschen Bucht jedoch sind bei Jungfischuntersuchungen nur Tiere ab $4 \mathrm{~cm}$ Totallänge nachgewiesen worden (Thielemann 1916, Bückmann 1934). Bislang fehlen hier Untersuchungen über das erste Auftreten der frühen Bodenstadien.

Die im folgenden dargestellten Laborbeobachtungen sollen zum weiteren Verständnis der Ethologie und Ökologie der Seezungenbrut beitragen und durch den Versuch, die Ergebnisse auf die Verhältnisse in See zu übertragen, Wege zu neuen Hypothesen über den Verbleib der älteren Larven aufzeigen.

\section{BEOBACHTUNGSMETHODIK UND LABORBEDINGUNGEN}

Das zur Erbrütung verwendete Eimaterial stammte von einer Anzahl Seezungen zwischen 30 und $37 \mathrm{~cm}$ Totallänge, die in einem $4 \mathrm{~m}^{3}$ großen Hälterungsbecken bei $12^{\circ} \mathrm{C}$ ablaichten (vgl. Flüchter 1965). Die Beobachtungen wurden nach Überführung aus den Erbrütungsanlagen bei einer Hälterungstemperatur von 14 bis $15^{\circ} \mathrm{C}$ sowohl in kleinen küvettenartigen Aquarien (Abmessungen $30 \times 20 \times 3 \mathrm{~cm}$ ) als auch in größeren Vollglasbecken mit Innenfilter (FLüchter 1964) durchgeführt. Die Dauer der Aktivitätsphasen wurde vom Dottersackstadium an bis zum Zeitpunkt der ersten Nahrungsaufnahme auf einem Kymographen über einen Zeitraum von jeweils 5 Minuten $z u$ verschiedenen Tageszeiten quantitativ registriert. Durch Betätigung eines elektrischen Kontaktes zeichnete der Beobachter auf einem mit konstanter Geschwindigkeit am Schreibhebel vorbeigeführten Wachsschichtpapierstreifen die Aktivitätszeiten in chronologischer Reihenfolge auf. Qualitative Beobachtungen wurden auf Tonband protokolliert. Bei den älteren Larvenstadien konnten die Aktivitätsphasen auf Grund der Schnelligkeit und Vielseitigkeit der Verhaltensweisen nicht mehr quantitativ erfaßt werden. Durch Einzelbildauswertungen von Filmaufnahmen wurden Lokomotionsmodus und Schwimmgeschwindigkeit ermittelt. Die Aufnahmen erfolgten bei unterschiedlichen Lichtintensitäten und verschiedenen Lichteinfallswinkeln, um den möglichen Einfluß des Lichtfaktors auf die Verhaltensreaktionen zu prüfen. Dabei wurde sowohl unter direktem Sonnenlicht als auch bei seitlich einfallendem Kunstlicht gearbeitet. Während die Filmaufnahmen ausschließlich in stehendem Wasser durchgeführt wurden, fanden Direktbeobachtungen auch bei leicht bewegtem Wasser statt. Für die Filmaufnahmen und Direktbeobachtungen der Dottersacklarven und der älteren Bodenstadien standen jeweils etwa 50 bis 100 Larven zur Verfügung.

\section{SCHWIMM- UND FRESSVERHALTEN DER LARVEN IM AQUARIUM}

Schwimmverhalten der frischgeschlüpften Dottersacklarven

Die Seezungenlarven schlüpfen mit einem auffallend großen Dottersack, der ihnen ein plumpes Aussehen verleiht (Abb. 1). Unmittelbar nach dem Schlüpfen schwimmen die etwa 2,6 bis 3,0 mm langen Dottersacklarven mit kurzen Stößen vorwärts. Sie legen häufig lange Ruhepausen ein. Infolge des spezifisch leichteren Dottersackes 
haben sie im Wasser leichten Auftrieb und treiben meist in Rückenlage. Häufig erreichen sie in dieser Haltung die Oberfläche (Abb. 1a). Ihre Schwimmrichtung ist in der Regel abwärts gerichtet. Die Bewegungen wirken infolge des ungünstigen Verhältnisses zwischen Dottersackgröße und Larvenlänge sehr unbeholfen und sind weitgehend ungesteuert. Im Gegensatz zum Schwimmverhalten anderer mariner Fischlarven, etwa der Heringsłarven, stellt hier die Bewegung keine über die Körperachse
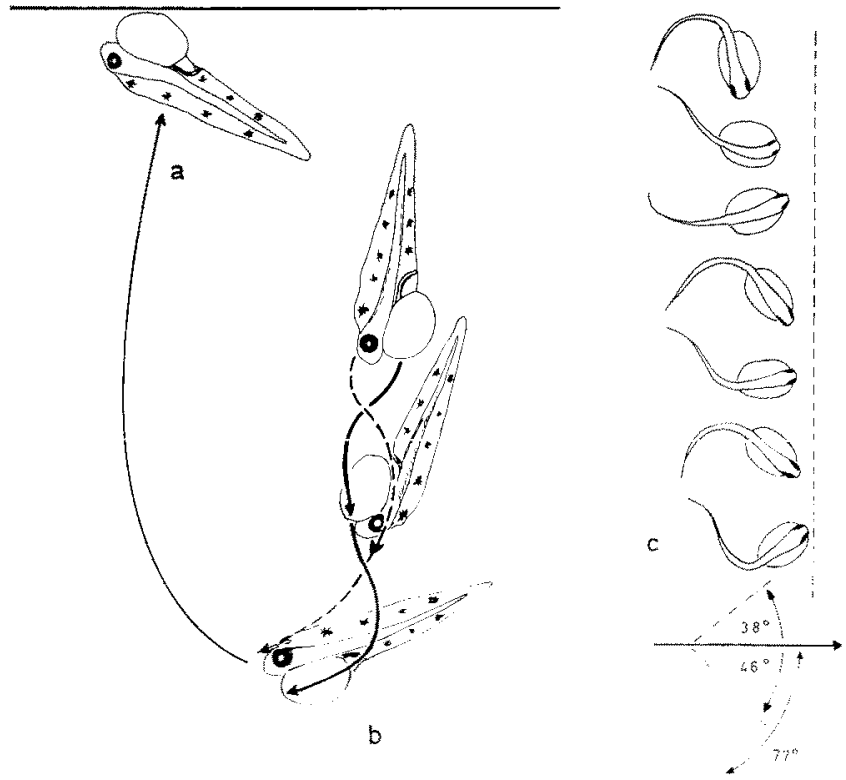

Abb. 1. Schwimmverhalten der Dottersacklarven von Solea solea (schematisch nach Filmaufnahmen; $a$ und b Seitenansicht, $c$ Aufsicht). a: Ruhelage der frischgeschlüpten Larven, welche in stehendem Wasser sehr oft bis an die Oberfläche treiben. b: Abwärtsgerichtete Schwimmbewegung mit Drehung um die Körperlängsachse. c: Schwanzschlagamplitude und Vortrieb im Wasser. Die gestrichelte Linie steht senkrecht zur Schwimmrichtung und stellt die Bezugslinie für die Vorwärtsbewegung dar. Der Laufbildfolge im Film entspricht die Bildfolge von oben nach unten. Aufnahmefrequenz. 24 Bilder pro Sekunde

sinusförmig verlaufende Wellenbewegung dar. Vielmehr erscheint der vordere Körperabschnitt durch den eng anliegenden Dottersack starr, während der flexible kaudale Teil um den Mittelpunkt der Körperlängsachse Schwingungen ausführt (Abb. 1c). Dabei wird der starre vordere Teil des Körpers unter einem Winkel von 40 bis $45^{\circ}$ (maximal 70 bis $80^{\circ}$ ) aus der Schwimmrichtung herausgedreht.

Während der ersten Lebenstage drehen sich die Larven beim Schwimmen häufig um ihre Körperlängsachse, so daß der Eindruck eines „spiraligen Vorwärtsschraubens“ entsteht (Abb. 1b). Generell erfolgt die Kraftübertragung auf den Dottersack tangential, und entsprechend der Größe der Schwanzschlagamplitude tritt bei allen Dottersacklarven ein mehr oder weniger deutliches "Taumeln" um die Körperlängsachse auf. $\mathrm{Da}$ bei den Seezungenlarven der Schwanzschlag häufig etwas einseitig betont verläuft, sind die Ausschläge der "Taumelbewegung" nach einer Seite hin größer und addieren 
sich während zahlreicher, kurz aufeinander folgender Schwanzschläge zu einer Drehbewegung um die Körperachse. Die Larven werden dabei durch wechselnde Lichtbedingungen nicht beeinflußt, wie durch wiederholte Versuche gezeigt werden konnte. Die Drehbewegungen werden auch nicht durch Seitenlicht induziert wie beispielsweise bei den Jungfischen von Pterophyllum scalare (v. Holst 1948, Mxtrelstaedt 1951). Oft sind die Aktivitätsphasen auch so kurz, daß aus der in Ruhepausen eingenommenen Rückenlage keine vollständige Aufrichtung zur aufrechten Körperhaltung (Bauchlage) erreicht werden kann. Die Larven treiben dann für kurze Zeit in Seitenlage, um bald wieder in die Ausgangslage zurückzukehren, falls die Aufrichtung nicht doch noch durch einen erneuten Schwimmstoß abgeschlossen wird. Weitere Schwimmstöße führen dann zu dem oben beschriebenen "spiraligen Vorwätsschrauben". Von Zeit zu Zeit schwimmen die Larven auch über längere Aktivitätsphasen in der normalen Körperlage (Bauchlage). In leicht bewegtem Wasser zeigen sie keine gesteigerte Aktivität und lassen sich in jeder Körperlage treiben.

\section{Verhalten zum Zeitpunkt der ersten Nahrungsaufnahme}

\section{Schwimmverhalten}

Mit fortschreitender Dotterzehrung verringert sich die Größe des Auftriebs rasch. Die Larven können sich schon am zweiten Tag nach dem Schlüpfen (bei $15^{\circ} \mathrm{C}$ ) freischwebend im Wasser halten. Dementsprechend wird auch die normale Körperhaltung (Bauchlage) häufiger eingenommen, obwohl noch immer Drehungen um die Körperachse beobachtet werden können. Die bevorzugte Schwimmrichtung nach unten wird jedoch beibehalten. Die Folge hiervon ist, daß sich die Mehrzahl der Larven vom dritten Tage an in Bodennähe des Aquariums aufhält. Hier stellen sich die Larven sehr bald auf eine optisch gegliederte Umwelt ein und ändern ihr Schwimmverhalten. In aufrechter Körperhaltung schwimmen sie mit leicht angewinkeltem Schwanzende dicht über dem Grund (Abb. 2a). Dabei ändern sie fortwährend ihre Schwimmrichtung, halten oft kurzfristig ein und biegen ihren Körper S-förmig oder U-förmig. Sehr häufig schwimmen sie auch rückwärts, ohne dabei die vorher eingenommene Körperhaltung (S-Form, U-Form oder leicht angewinkeltes Schwanzende) aufzugeben.

Die Schwanzschlagamplitude der nunmehr etwa $4 \mathrm{~mm}$ langen Larven ist nur noch gering. Oft unterbleibt der Schwanzschlag völlig, und die Fortbewegung sowie die Steuerung der Bewegungsrichtung und Körperhaltung erfolgt im wesentlichen durch den intensiven Einsatz der bereits gut ausgebildeten Pectoralflossen. Demgegenüber kann auch der Schwanzschlag im Zusammenwirken mit den Brustflossen oder auch allein kurzfristig so intensiv werden, daß ein besonders schnelles Vorwärtsschwimmen möglich wird. Dies ist am häufigsten dann zu beobachten, wenn die Larven vorübergehend den Boden verlassen und in freies Wasser aufsteigen.

Während der Ruhepausen, die in immer unregelmäßigeren Abständen auftreten und von sehr unterschiedlicher Dauer sein können, stellen sich die Larven zunächst in aufrechter Haltung auf den Grund und neigen sich oft nach einiger Zeit geringfügig zur einen oder anderen Körperseite hin, wobei das Schwanzende regelmäßig vom 
Boden abgewandt wird (Abb. 2c). Gelegentlich kippen sie auch ganz um und bleiben schon wenige Tage nach der ersten Nahrungsaufnahme vorübergehend in Seitenlage gestreckt am Boden liegen. Deutlich ausgeprägt ist dies Verhalten jedoch erst bei etwas älteren Larven (5-7 mm Totallänge). Grundsätzlich wird beim Hinlegen keine Körperseite bevorzugt. Das Kippen zur einen oder anderen Seite hin ist zufallsmäßig verteilt. Es konnte sogar oft ein Umdrehen von einer Körperseite auf die andere in beiden Richtungen beobachtet werden. Erst kurz vor der Metamorphose erfolgt das Umdrehen von der einen zur anderen Körperseite meistens nur noch dann, wenn die Larve nicht auf die spätere Blindseite zu liegen kommt.

Gleichzeitig mit dem Einsetzen dieser Verhaltensweisen treten andere Schwimmbewegungen auf, die funktionell in engem Zusammenhang mit der Nahrungsaufnahme stehen.
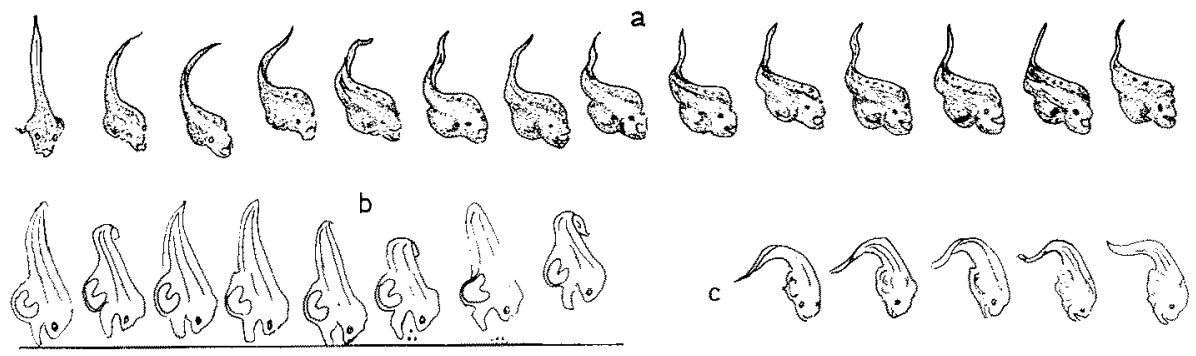

Abb. 2: Schwimmverhalten von Seczungenlarven zum Zeitpunkt der ersten Nabrungsaufnahme (umgereichnet nach Fimaufrahmen; de Bildfolge entsprich der Iaufbildfolge im Film, Aufnahmefrequen\% 24 Bilder pro Sckunde) a: Shwimmen mit leidt angewinkeltem Schwanzende dich über dem Grund. b: Erste Nabrungsaufname am Boden. c. Seitwärtsneigen und Hinlegen am Boden mit Aufbiegen der Schwanzregion

\section{Erste Nahrungsaufnahme}

Die nun in Bodennähe lebenden Seezungenlarven beginnen mit der aktiven Nahrungsaufnahme (3. und 4. Tag nach dem Schlüpfen; etwa 4 bis $4,5 \mathrm{~mm}$ Totallänge; Abb. 3). Sic schwimmen dabei zunächst senkrecht gegen den Untergrund. Die Schwimmstöße sind kurz und kräftig. Unter Linkrümmung des Schwanzendes und regem Einsatz der Brustflossen heben sie sich rückwärtsschwimmend wieder vom Boden ab, um mit erneutem Anlauf das Maul in den Boden zu stoßen (Abb. 2b). Mehr und mehr beginnen die Larven ihr Maul vor dem Zustoßen zum Boden weit aufzusperren. Je nach Art des gebotenen Substrats gelingt es den Larven, Partikel unterschiedlicher Größe vom Grunde aufzunehmen und nach Nahrungsorganismen abzusuchen. Sandkörnchen und Detritusteilchen werden nach sorgfältiger Prüfung im Maul wieder ausgespuckt. Bietet man den Larven keinen Sand auf dem Aquarienboden, so zeigt die Mehrzahl der Larven das gleiche Verbalten im Leerlauf. Hungrige Tiere jagen auch im freien Wasser nach Planktern, ein Verhalten, welches man bei satten Larven seltener beobachten kann. Bei einem reichlichen Angebot von Harpacticiden war der Erfolg der Beutefanghandlungen gering. 
Bei der aktiven Nahrungsaufnahme im freien Wasser unterscheiden sich Seezungenlarven von anderen marinen Fischlarven vor allem durch die fehlende Zielstrebigkeit der Nahrungssuche. Steigen die Larven vom Grunde auf und kommen ihnen Artemia salina-Nauplien unmittelbar in das Gesichtsfeld (Wahrnehmungsabstand bei Kunstlicht 500 Lux etwa 3 bis $4 \mathrm{~mm}$ ), so schwimmen sie zunächst unschlüssig von einem Nauplius zum anderen. Sehr bald beginnen sie jedoch heftig zu schnappen. Das gleiche Verhalten beobachtet man bei Fütterung mit Wildplankton. Nur selten sind die Larven bei diesem Beutefang erfolgreich. Oft schnappen sie schon $\mathrm{zu}$, wenn sie noch 1 bis $2 \mathrm{~mm}$ vom Beutetier entfernt sind. Auffallend ist, daß die

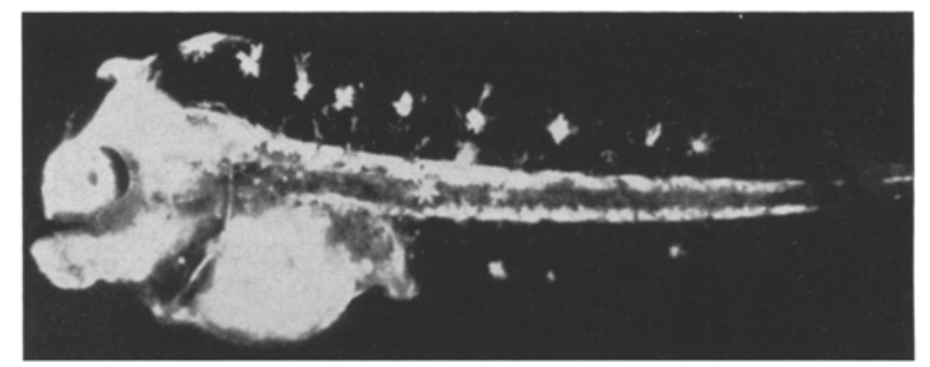

Abb. 3: Seezungenlarve zum Zeitpunkt der ersten Nahrungsaufnahme (Dunkelfeldaufnahme). Auffallend ist das für eine Firschlarve sehr große Maul

Beutefanghandlungen der Seezungenlarven im Gegensatz zum Beuteerwerb vieler anderer Fischlarven (BRAUM 1963, 1964) in der Regel nicht mit einer Einstellreaktion (S-förmige Körperhaltung) eingeleitet wird. Die meisten der beobachteten erfolgreichen Beutefanghandlungen beruhen auf Zufallstreffern. Lediglich ein geringes aber kräftiges Vorwärtsschwimmen bringt die Larve in die Nähe des Beutetieres. Dabei erinnert das Schwimmverhalten sehr stark an den Bewegungsmodus zur Nahrungsaufnahme vom Substrat.

\section{Verhalten der älteren Bodenstadien vor und während der Metamorphose}

Die älteren symmetrischen Seezungenlarven ( 7 bis $7,5 \mathrm{~mm}$ Totallänge) zeigen neben den geschilderten Verhaltensreaktionen zum Zeitpunkt der ersten Nahrungsaufnahme bereits zahlreiche Verhaltensweisen der späteren metamorphosierten Jungtiere. Vorwiegend schwimmen sie in Schräg- oder Seitenlage (Abb. 4) und nehmen zeitweise den Lokomotionsmodus der adulten Tiere an. Die Pectoralflossen, welche gegenüber der Dorsalen und Analen nicht mehr merklich weitergewachsen sind, verlieren mit fortschreitender Entwicklung mehr und mehr ihre Wirksamkeit. Schon mit einsetzender Metamorphose wirken Dorsale und Anale funktionell als Flossensaum, wenngleich auch die gesamte Körperachse vorerst in die Wellenbewegungen einbezogen bleibt. Nach der Metamorphose hat der Körper keinen Anteil an den Undulationen der Flossen, deren Tätigkeit synchron, gegenläufig oder auch nur einseitig verlaufen 
können. Die Bewegungsweise der adulten Tiere beschreibt in gleicher Weise v. WAHLERT (1961) ausführlich und macht auf die Ausbildung eines einheitlichen Flossensaumes durch Reduktion der Paarflossen sowie Reduktion der Höhe der Unpaarflossen unter Einbeziehung der Kaudalen aufmerksam.

Noch vor der Augenwanderung sind die Larven fähig, sich an das Substrat anzuklammern. Dabei drücken sie entweder die Kopfpartie in eine Bodenmulde und

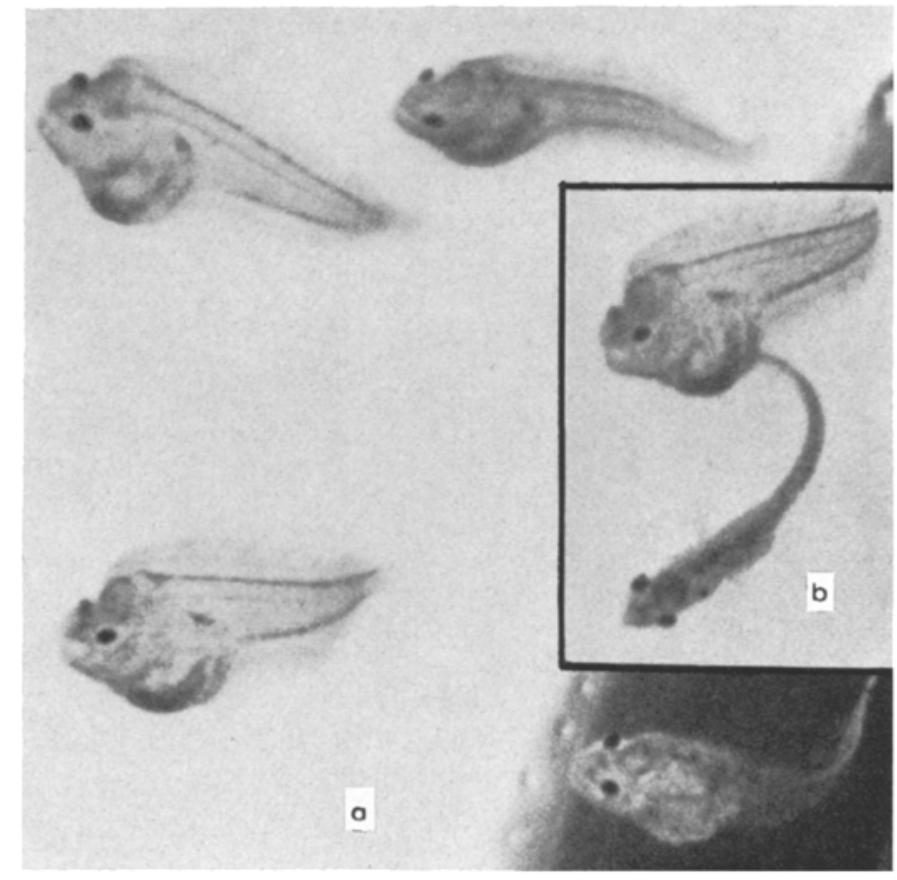

Abb, 4: Angefütterte, noch symmetrische Seezungenbrut kurz vor Beginn der Augenwanderung (Einzelbilder aus Filmaufnahmen). a: In Schräg- und Seitenlage schwimmend. b: Untere Larve in aufrechter Haltung mit U-förmig gebogenem Schwanzende. Deutlich ist an der Augenstellung zu erkennen, daß dic Larve noch völlig symmetrisch ist

stützen sich mit leicht angewinkeltem Schwanz auf die Kaudalflosse, oder sie wölben sich kuppelförmig auf, den Flossensaum, Kopf und Schwanz dabei eng an den Boden anlegend. Bei schnellem Aufschwimmen, wie es beispielsweise durch Anstoßen mit einem Glasstab bewirkt werden kann, ist ein dem Omega-Sprung ähnliches Verhalten zu beobachten, wic es Kruvg (1963) bei adulten Tieren beschreibt. Das Eingraben in den Sand konnte jedoch nicht vor beendeter Metamorphose beobachtet werden. Auch eine direkte Farbanpassung an hellen Untergrund erfolgte selbst bei längerer Expositionsdauer nicht. Dagegen änderte sich mit zunehmendem Alter die Körperfärbung mehrmals. Die im Laufe der Larvalentwicklung wechselnde Färbung auf Grund von Anderungen in der Pigmentierung beschreibt FLÜCHTER (1965) ausführlich und weist auf die mögliche Bedeutung zur Unterscheidung von Alters- und Entwicklungsstadien hin. 
Bereits einige Tage vor der Metamorphose werden die Larven weitgehend inaktiv. Sie schwimmen nur noch selten im freien Wasser und nehmen ihre Nahrung fast ausschließlich vom Boden her auf. Schließlich stellen sie während der Metamorphose die Nahrungsaufnahme ein.

\section{SCHWIMMLEISTUNG DER DOTTERSACKLARVEN}

Trotz hoher Schwanzschlagfrequenz und of großer Schwanzschlagamplitude erscheint der Vortrieb im Wasser bei Dottersacklarven im Verhältnis zum Arbeitsaufwand gering. Hierfür gibt die Bremswirkung des großen Dottersacks eine durchaus

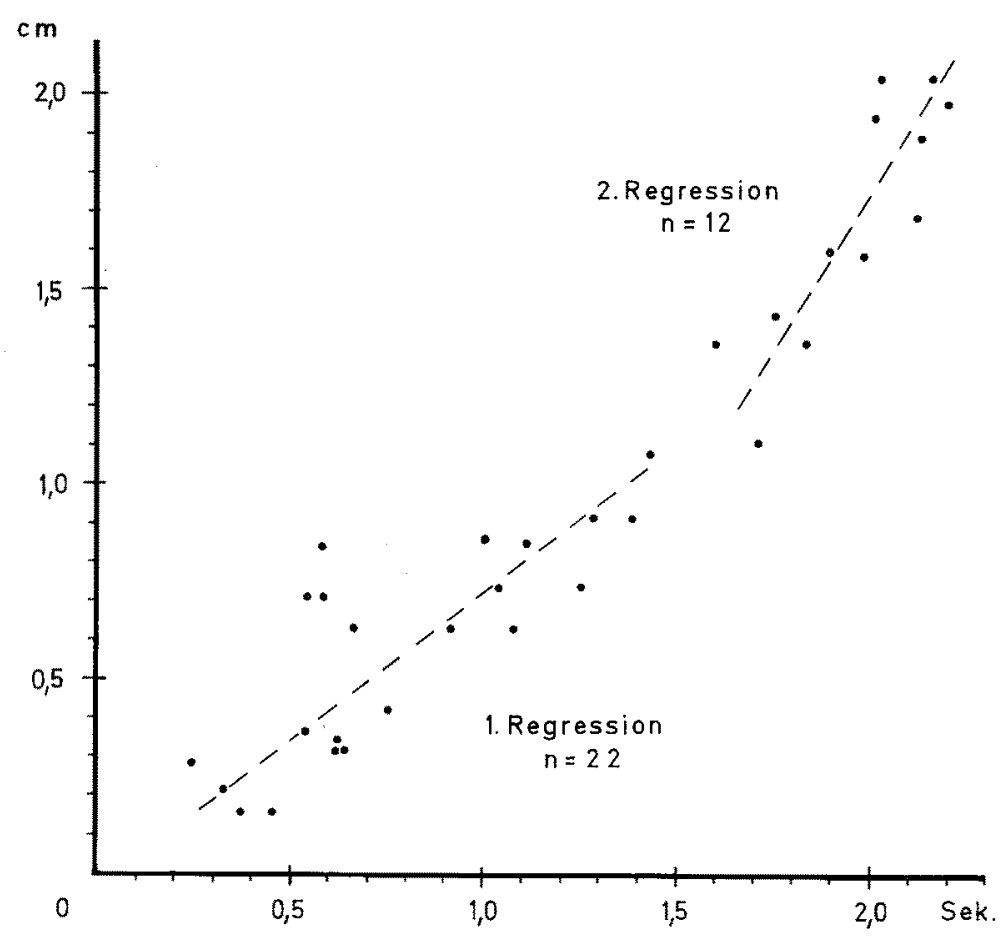

Abb. 5: Weg-Zeit-Kurve für kurze Schwimmstrecken der Dottersacklarven. Bei den relativ seltenen längeren Schwimmphasen werden höhere Geschwindigkeiten erreicht (nach Filmaufnahmen, Aufnahmefrequenz 24 Bilder/Sekunde)

hinreichende Erklärung. Darüber hinaus muß berücksichtigt werden, daß die Kaudalflosse noch völlig unentwickelt ist. Gegen Ende des Dottersackstadiums vermag die beginnende Ausbildung der Hypuralia die Fortbewegung wirksam zu unterstützen. Die Dauer der einzelnen Schwimmstöße liegt in der Regel zwischen 0,3 und 1,5 Sekunden. Es werden dabei Durchschnittsgeschwindigkeiten von etwa 6 bis $9 \mathrm{~mm} / \mathrm{sec}$ erzielt. Geschwindigkeiten also, die zwei bis drei Larvenlängen pro Sekunde durchaus erreichen. Wie die Messungen aus Filmaufnahmen zeigen (Abb. 5) streuen die Werte der Weg- 
Zeitkurve für die kurzen Aktivitätsphasen (etwa 0,5 bis 0,7 sec Dauer) erheblich (1. Regression). Sind die Aktivitätsphasen länger, dann steigt auch die Durchschnittsgeschwindigkeit (2. Regression).

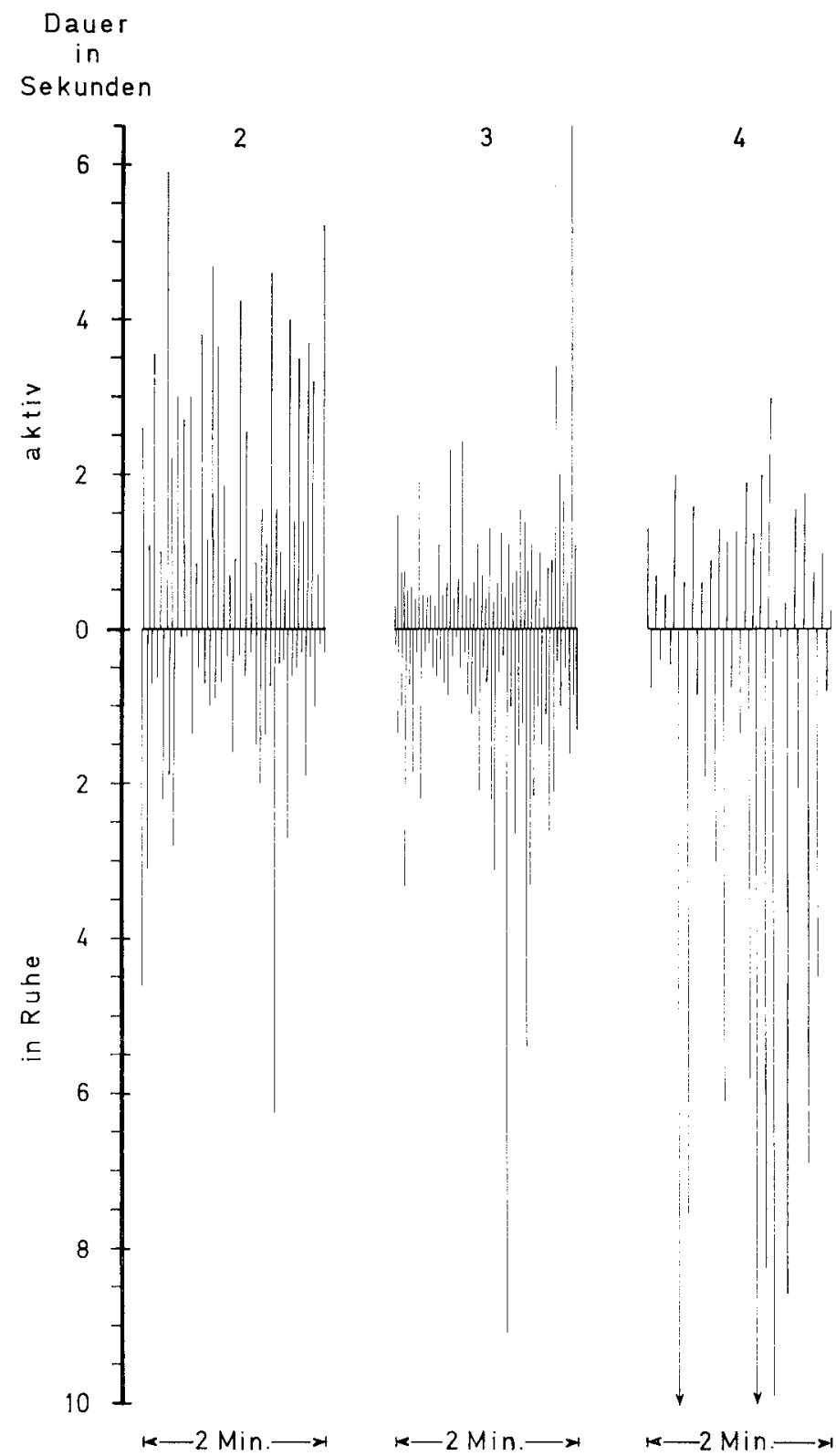

Abb. 6: Chronologische Folge (von links nach rechts) und Dauer der Aktivitäts- und Ruhephasen während der ersten beiden Beobachtungsminuten der Protokolle 2, 3 und 4 


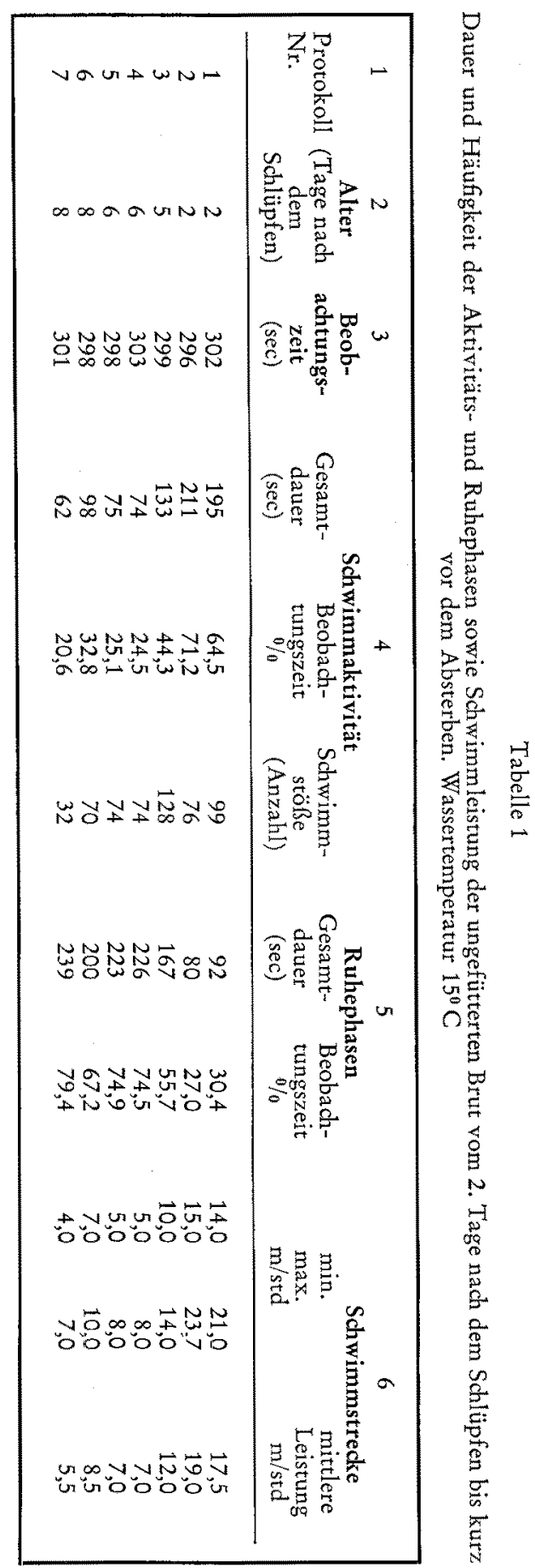


Diese Tatsache kann wie folgt erklärt werden: In ihrer Unbeholfenheit überwinden die Larven den Anfangswiderstand bei Beginn der Schwimmbewegungen unterschiedlich, so daß eine starke Streuung der Schwimmgeschwindigkeit während der kurzen Aktivitätsphasen hervorgerufen wird. Um zu erfahren, wie groß die tägliche

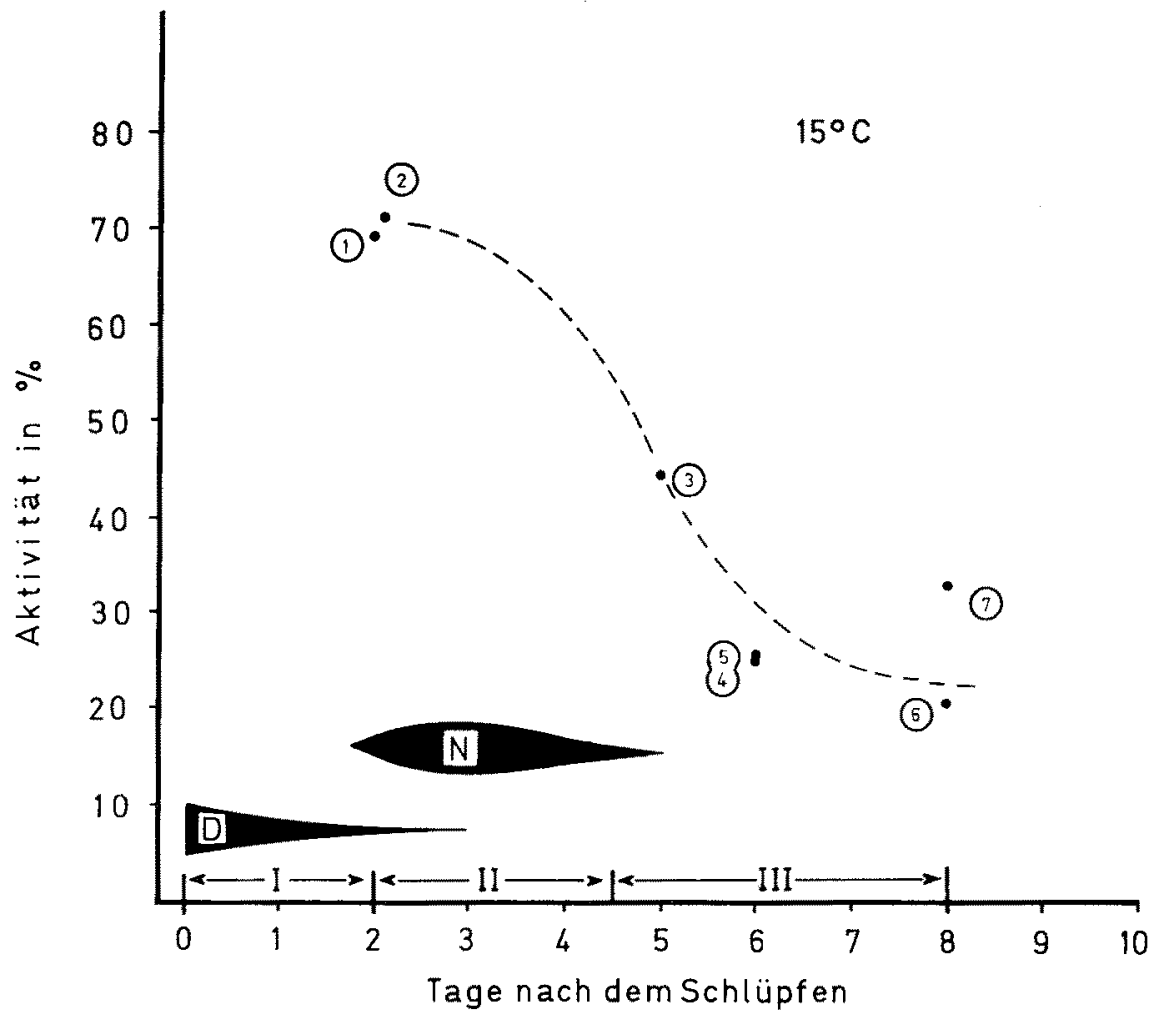

Abb. 7: Aktivitätsabnahme der ungefütterten Larven bei $15^{\circ} \mathrm{C}$ vom 2. Tage nach dem Schlüpfen bis kurz vor dem Absterben. Gesamtdauer der Aktivitätsphasen dargestellt in Prozent der Gesamtbeobachtungszeit (nach Daten der Tabelle 1, Protokoll-Nr. 1-7). D: Verlauf der Dotterzehrung; N: Zeitraum, in dem Larven bei Plankton-Angebot Nahrung aufnehmen würden. Beginn und Erlöschen der Beutefanghandlungen. I: Dottersadkstadium; II: Zeit, in der die Larven bei Planktonfütterung angefütter werden kömnen; III: Larven zeigen bei Angebot von Plankton kein Interesse mehr für die Nahrung und sterben langsam ab

Schwimmstrecke gegen Ende des Dottersackstadiums ist, muß man die Häufigkeitsverteilung und Dauer der Aktivitätsphasen über einen längeren Zeitraum erfassen und die erhaltenen Werte mit Hilfe der Daten der Weg-Zeitkurve in die entsprechende durchschnittliche Schwimmstrecke umrechnen. Die Aktivitätsverteilung konnte mit Hilfe des eingangs erwähnten Kymographen ermittelt werden.

In Tabelle 1 geben die Protokolle 1 und 2, die hier zunächst betrachtet werden sollen, die typische Aktivitätsverteilung und -dauer zum Zeitpunkt der ersten Nahrungsaufnahme wieder. Die Beobachtungszeit beträgt jeweils 5 Minuten. In Spalte 4 der Tabelle ergibt sich aus der Anzahl der beobachteten Schwimmstöße und der Ge- 
samtaktivitätsdauer eine durchschnittliche Dauer der einzelnen Schwimmstöße von etwa 1,9 bis 2,7 Sekunden. Für die jüngeren Dottersacklarven ließ sich aber aus den Filmaufnahmen nur eine um vieles geringere durchschnittliche Dauer der Schwimmbewegungen bestimmen. Die Ursache für diesen Unterschied ist im Auftreten einiger sehr langer Aktivitätsphasen zur Zeit der ersten Nahrungsaufnahme zu suchen. Da die kurzen und langen Aktivitätsphasen zufallsmäßig auf die Beobachtungszeit verteilt sind (Abb. 6), sind zur Berechnung einer mittleren Schwimmleistung beide aus Filmaufnahmen gemachten Schätzungen (1. u. 2. Regression) zu berücksichtigen. In Spalte 6 der Tabelle 1 sind die minimalen und maximalen sowie die Durchschnittsleistungen zusammengestellt. Danach liegt die Schwimmleistung zum Zeitpunkt der ersten Nahrungsaufnahme unter den herrschenden Versuchsbedingungen zwischen 17 und $19 \mathrm{~m} / \mathrm{h}$.

Auf Grund der vorliegenden Beobachtungen und Meßergebnisse läßt sich eine tägliche Schwimmstrecke zum Zeitpunkt der ersten Nahrungsaufnahme unter Berücksichtigung eines $24-S t u n d e n-T a g e s$ mit 400 bis $460 \mathrm{~m}$ angeben. Unberücksichtigt bleibt, ob die jungen Seezungenlarven bereits einer Tagesrhythmik unterliegen. Da die individuelle Leistung stark variiert, könnte eine mögliche Periodizität nur an wesentlich umfangreicheren Beobachtungen nachgewiesen werden.

Ungefütterte Seezungenlarven lassen sehr bald in ihrer Schwimmaktivität nach; ihre Leistungen nehmen rasch ab (Tabelle 1, Protokolle 1 bis 7). Es überwiegen zunehmend die kurzen Schwimmstöße (Abb. 6, Nr. 3). Am 8. Tage nach dem Schlüpfen schwimmen ungefütterte Larven kaum mehr als 4 bis $7 \mathrm{~m} / \mathrm{h}$.

In Abbildung 7 sind die Werte der Tabelle 1 graphisch dargestellt. Gibt man die Gesamtdauer der Aktivitätsphasen in Prozent der Gesamtbeobachtungszeit an, so beträgt sie am zweiten Tag nach dem Schlüpfen etwa $70 \%$. Zu diesem Zeitpunkt setzen bei Angebot von Plankton die Fanghandlungen ein. Larven, denen bis zum 5. Tage nach dem Schlïpfen kein Plankton angeboten wird, sind nur noch bis zu 50\% der Gesantbeobachtungszeit aktiv. Vom 6. Tage an ist es nicht mehr möglich, die Larven anzufüttern. Trotz reichlichem Angebot von Artemia-Nauplien und Wildplankton konnten unter den gegebenen Versuchsbedingungen keine Beutefanghandlungen mehr beobachtet werden.

\section{DISKUSSION}

Auf Grund der mitgeteilten Beobachtungen müssen einige unserer bisherigen Vor-stellungen über die Naturgeschichte der Seezungenbrut revidiert werden. Das pelagische Larvenstadium ist auffallend kurz und fast ausschließlich auf das Dottersackstadium beschränkt. Da die Laborbedingungen von den natürlichen Verhälnissen abweichen, wird die Frage nach der Größe des Auftriebs der Dottersacklarven in Abhängigkeit von der Erbrütungstemperatur sowie der Einfluß von Strömungen auf die Verhaltensreaktionen im freien Wasser bedeutsam sein können. Allgemein wird angenommen, daß Fischlarven, die bei hohen Wassertemperaturen erbrütet wurden, einen größeren Dottervorrat haben als solche, die bei niederen Erbrütungstemperaturen schlïpfen. Dementsprechend wäre zu erwarten, daß der Auftrieb der in See bei 4 bis $5^{\circ} \mathrm{C}$ niedrigeren Temperaturen geschlüptten Brut bedeutend geringer ist, als bei 
den Versuchstieren. Allerdings besitzt dieses Wasser eine höhere Dichte, was wiederum den Auftrieb vergrößern würde. Nehmen wir aber an, daß der Auftrieb der in See geschlüpften Brut wirklich geringer ist, so würde dies zur Folge haben, daß die Larven bei bevorzugt abwärtsgerichteter Schwimmbewegung den Grund noch schneller erreichen, als sich aus den Meßwerten der Beobachtungsprotokolle abschätzen läßt.

Sicher wird die Larve nicht den direkten Weg von der Oberflächennähe zum Boden hin einschlagen, zumal unter natürlichen Bedingungen der Einfluß wechselnder Strömungen und Turbulenzen ständige Veränderungen der Körperlage aufzwingen und so möglicherweise die pelagische Lebensweise verlängern. Fabre-Domergue \& Brérrix (1905) nehmen eine sehr ausgedehnte pelagische Lebenszeit an. Es ist aber gut möglich, daß sich die an die Seitenwände der Erbrütungsgläser anklammernden Larven nur gegen die durch Rührwerke erzeugte Kreisströmung einstellten und nicht eigentlich pelagisch waren. Selbst wenn durch wechselnde Umwelteinflüsse die Larven zeitweilig von der abwärtsweisenden Schwimmrichtung abgelenkt werden, dürften sie doch gegen Ende des Dottersackstadiums in wenigen Stunden, höchstens Tagen den Boden in den flacheren Teilen der Nordsee erreichen. Die Larven werden zumindest vom Zeitpunkt der ersten Nahrungsaufnahme an bestrebt sein, den Boden aufzusuchen. Wie aus den Versuchen zur Aktivitätsabnahme der ungefütterten Brut hervorgeht, können diejenigen Larven, die bis zum 5. Tag nach dem Schlüpfen nicht zum Fressen gekommen sind, aus dem Plankton kaum noch Nahrung aufnehmen. Bei den im freien Wasser herrschenden niedrigeren Temperaturen wird dieser Zeitraum sicher um 1 bis 3 Tage länger sein. Es bleibt zu prüfen, ob die Larven dann noch in der Lage sind, Nahrung vom Boden her aufzunehmen.

Das bei Seezungenlarven häufig auftretende Schwimmen in spiraligen Bahnen kann sicherlich nicht auf ein zu frühes Schlüpfen zurückgeführt werden: Stintlarven (nach mündlicher Mitteilung von Dr. Lillelund) und Heringslarven (eigene Beobachtung) zeigen solch Verhalten, wenn man sie bei zu hohen Temperaturen vor zeitig schliipfen läßt. Bei den Seczungenlarven kann aber ein derartiges Verhalten auch noch nach dem Dottersackstadium gelegentlich beobachtet werden. In einem Hälterungsbecken wurden im Frühjahr 1965 zur Streckung des Eimaterials auch Erbrütungen bei $9^{*}$ bis $10^{\circ} \mathrm{C}$ durchgeführt. Die bei diesen Temperaturen geschlüpften Larven zeigen kein abweichendes Verhalten und man darf annehmen, daß das zeitweilige Schwimmen in spiraligen Bahnen zum natürlichen Verhaltensspektrum der Seezungenbrut gehört.

Da die Seezungenlarven schon zum Zeitpunkt der ersten Nahrungsaufnahme das Substrat abweiden können und nicht eigentlich reine Planktonfresser sind ${ }^{1}$, wird für eine Beurteilung der Überlebenschancen der Brut nicht nur der Zeitpunkt, sondern auch der Ort, an welchem die Larven den Boden erreichen, wichtig sein. Zeitpunkt und Ort des Auftretens der Seezungenbrut am Grund sind stark von den herrschenden Umweltbedingungen abhängig. So hat die Wassertemperatur bekanntlich großen Einfluß auf die Laichaktivität sowie den Beginn der Laichzeit. Die in Lake Quarun (Ägypten;

1 Die hohe Uberlebensrate bei der Aufzucht mit Artemia-Nauplien ist wahrscheinlich auf dic außergewohnlich hohe Naupliendichte zurükzuführen (vgl. Fü̈cHTER 1965), zumal die Nauplien, dem Lichteinfluß folgend, sich an bestimmten Stellen des Aquariums in Bodennähe besonders eng zusammenballten. 
vgl. EL-SARKA 1963) eingebürgerten Seezungen waren bereits im Januar reif und hatten Mitte Februar bis Ende März abgelaicht. Die Luftemperaturen liegen dort im Januarmittel bei $17,6^{\circ}$ und im Juli bei etwa $34,2^{\circ} \mathrm{C}$. In der Nordsee laicht die Seezunge im Mai bis Juni bei Wassertemperaturen zwischen $9^{\circ}$ und $11^{\circ} \mathrm{C}$.

Hohe Temperaturen begünstigen die Laichaktivität und beschleunigen die Entwicklungsgeschwindigkeit der Eier und Larven. Der Zeitraum, in dem die Brut von Jahr zu Jahr den Strömungen ausgesetzt ist, kann beträchtlich schwanken. Ebenso können Anderungen der Strömungsverhältnisse eine Verdriftung in ungünstige Gebiete bewirken. Es wäre wichtig zu wissen, welche Substratbedingungen mit welcher Bodenfauna für die erste Nahrungsaufnahme am günstigsten sind. Ist eventuell das $\mathrm{Ab}$ weiden größerer Steinblöcke und festen Untergrundes möglich? Bietet ein Schlickboden in ruhigem Wasser ideale Aufwuchschancen? Wie stark darf die Strömung sein, damit die Brut sich am Grunde halten und vom Substrat fressen kann? Zur Beantwortung dieser Fragen wären zahlreiche experimentell-ökologische Untersuchungen erforderlich. Die exsten Experimente hierzu werden zur Zeit von Flüchter auf Helgoland durchgeführt.

\section{ZUSAMMENFASSUNG}

1. Die Dottersacklarven von Solea solea L. schwimmen sehr unbeholfen und drehen sich während der Schwimmbewegungen häufig um ihre Körperlängsachse. In der Regel halten sie dabei eine abwärtsgerichtete Schwimmbewegung ein und haben in den Ruhepausen leichten Auftrieb, der mit zunehmender Dotterzehrung schwindet. Gegenüber geringen Strömungen verhalten sie sich passiv und lassen sich in jeder Körperlage treiben.

2. Zum Zeitpunkt der ersten Nahrungsaufnahme befinden sich die meisten Larven in Bodennähe und weiden als Substratfresser den Grund nach freßbaren Organismen $\mathrm{ab}$.

3. Die gelegentlichen Beutefanghandlungen im freien Wasser, die bei hungrigen Larven häufiger auftreten, unterscheiden sich erheblich von denen anderer Fischlarven. Vor allem fällt die fehlende Zielstrebigkeit auf. Die Mehrzahl der erfolgreichen Fanghandlungen beruht auf Zufallstreffern.

4. Bereits neun bis zehn Tage nach der ersten Nahrungsaufnahme beginnen sich die noch völlig symmetrischen Larven zeitweise seitwärts auf den Grund zu legen, wobei keine Körperseite bevorzugt wird. Ein Wechseln von einer zur anderen Körperseite ist oft $z u$ beobachten.

5. Die älteren symmetrischen Larven zeigen noch alle die unter Punkt 2 bis 4 genannten Verhaltensweisen. Darüber hinaus haben sie bereits die Fähigkeit, sich an das Substrat anzuklammern. Häufig schwimmen sie auch in Schräg- oder Seitenlage und verhalten sich zeitweise wie Jungfische.

6. Eingraben konnte vor der Metamorphose nicht beobachtet werden. Farbanpassung an hellen Untergrund scheint trotz längerer Expositionsdauer $(>1$ Tag) nicht möglich zu sein.

7. Die Messung der Schwimmleistung auf Grund von Filmaufnahmen ergibt eine 
Durchschnittsleistung von 6 bis $9 \mathrm{~mm} / \mathrm{sec}$. Dies gilt für Dottersacklarven bis zum Zeitpunkt der ersten Nahrungsaufnahme.

8. Die tägliche Schwimmstrecke verschieden alter, ungefütterter Dottersacklarven wurde auf Grund von Registrierungen der Schwimmaktivität für Beobachtungsintervalle von jeweils 5 min bestimmt. Sie liegt zum Zeitpunkt der ersten Nahrungsaufnahme unter Annahme eines 12-Stunden-Tages bei etwa 200 bis $230 \mathrm{~m}$.

9. Gegen Ende des Dottersackstadiums sind die Larven bis zu 75\% der Beobachtungszeit aktiv. Bei ungefütterten Larven sinkt die Aktivität am 5. Tag nach dem Schlïpfen auf unter 50\% der Beobachtungszeit herab. Unter den herrschenden Versuchsbedingungen $\left(15^{\circ} \mathrm{C}\right)$ wurden zu diesem Zeitpunkt die Fanghandlungen auf Plankter eingestellt. $\mathrm{Ob}$ dann noch eine Nahrungsaufnahme vom Boden her möglich ist, wurde nicht geprüft. Vom 8 . Tage nach dem Schlïpfen an sank die Schwimmstrecke auf eine Stundenleistung von 4 bis $7 \mathrm{~m}$ ab.

10. Aus den Befunden wird geschlossen, daß die pelagische Lebensweise im wesentlichen auf das Dottersadkstadium beschränkt ist. Für die Beurteilung der Uberlebenschancen wäre wichtig zu wissen, welches Substrat mit welcher Bodenfauna für die erste Nahrungsaufnahme unter natülichen Bedingungen am günstigsten ist.

Herrn Dr. J. FuÜchTFr danke ich herzlich für die Uberlassung der Eier und Larven sow ic für dic Unterstuit»ung bei der Durchführung der Versuche und Beobachtungen. Dem Direktor der Biologischen Anstalt Helgoland, Herrn Prof. Dr. O. Kinne, danke ich für die Bercitstellung eines Arbeitsplatzes. Die Arbeit wurde mit finanzieller Unterstützing der Deutschen Forschungsgemeinschaft durchgeführt.

\section{ZITIERTE LITERATUR}

Braum, E., 1963. Die ersten Beutefanghandlungen junger Blaufelchen und Hechte. Z. Tierpsychol. 20, 257-266.

- 1964. Experimentelle Untersuchungen zur ersten Nahrungsaufnahme und Biologie an Jungfischen von Blaufelchen (Coregonus wartmanni BLOCH), WeiBfelchen (Coregonus fera JuRINE) und Hechten (Esox lucius L.) Arch. Hydrobiol. (Suppl. Bd) 28 (5), 183-244.

BückmanN, A., 1934. Untersuchungen über die Naturgeschichte der Seezunge, die Seczungenbevölkerung und den Seczungenfang in der Nordsee. Ber. dt. wiss. KommnMeeresforsch. 7 , $49-114$.

Cunningham, J. T., 1890. A treatise on the common sole (Solea vulgaris), considered both as an organism and as a commodity. Mar. Biol. Ass., Plymouth, $147 \mathrm{pp}$.

Ehrinbaum, E., 1907. Uber Eier und Jugendformen der Seezunge und anderer im Frühjahr laichender Fische der Nordsee. Wiss. Meeresunters. N. F. Abt. Helgoland 8, 201-269.

- 1914. Die Seezunge (Solea vulgaris Quensel) in fischereilicher und biologischer Beziehung. Mitt. naturh. Mus. Hamb. 31, 367-390.

El-SARKa, S., 1963. Acclimatization of Solea onlgaris (Linn.) in Lake Quarun, Egypt. J. Cons. perm. int. Explor. Mer 28, 126-136.

HotST, E. voN, 1948. Quantitative Untersuchungen über Umstimmungsvorgänge am Zentralnervensystem. 1. Der Einfluß des "Appetits" auf das Gleichgewichtsverhalten von Pterophyllum. Z. vergl. Physiol, 31, 134-148.

Fabre-Domergue.P. \& Bítrix, E., 1905. Développement de la sole (Solea vulgaris). Vuibert \& Nony, Paris, 266 pp. (Trav. Lab. Zool. marit. Concarneau.) 
Fü̈CHTER, J., 1964. Eine besonders wirksame Aquarienfilterung und die Messung ihrer Leistung. Helgoländer wiss. Meeresunters, 11, 168-170.

- 1965. Versuche zur Brutaufzucht der Seezunge Solea solea in kleinen Aquarien. Helgoländer wiss. Meerestinters. 12, 395-403.

KRUUG, H., 1963. Diurnal periodicity in the activity of the common sole, Solea vulgaris Quensel. Neth. J. Sea Res. 2, 1-28.

MittelstaedT, H., 1951. Dunkeladaptation und Nachtreaktion bei Pterophyllum. Naturwissenschaften 38, 137-138.

ThrelemanN, M., 1916. Einige Beobachtungen über das Wachstum der Seezunge (Solea vulgaris QUENSEL) und ihr Vorkommen im Nordfriesischen Wattenmeer. Wiss. Meeresunters. N. F. Abt. Helgoland 11, 199-216.

Wahlert, G. von, 1961. Die Entstehung der Plattfsche durch ökologischen Funktionswechsel. Zool. yb. (Syst. Ökol. Geogr. Tiere) 89, 1-42. 